\title{
Traitements de matériaux métalliques et céramiques par laser à excimères
}

\author{
M. Autric, T. Sarnet et C. Prat \\ Institut de Mécanique des Fluides, Equipe Interaction Laser-Matière, Parc Scientifique et \\ Technologique de Luminy, 13009 Marseille, France
}

\begin{abstract}
Résumé : Les résultats présentés dans cette communication rendent compte d'expériences menées au moyen de laser à exciplexes exclusivement en vue de l'amélioration des propriétés d'usage de certains matériaux métalliques et céramiques utilisés dans l'industrie automobile, aéronautique ou de l'espace. Les principaux résultats concernent les modifications des caractéristiques mécaniques (rugosité, dureté, ténacité, champ de contraintes,...), physico-chimiques (composition de surface, microstructure,...) et électrique (conductivité).
\end{abstract}

L'utilisation de rayonnements laser pour la transformation et le traitement de surfaces de matériaux métalliques et céramiques à des fins industrielles a suscité depuis quelques années un grand intérêt tant au niveau de la compréhension fondamentale des processus mis en jeu que pour les avancées technologiques que cela a pu engendrer dans le domaine industriel.

Lors de l'irradiation d'échantillons à bas et moyen flux, les phénomènes rencontrés et les effets résultants sur le matériau sont de natures diverses et dépendent principalement de différents facteurs qu'il est possible de regrouper dans deux catégories:

- Les facteurs liés à la source laser et aux conditions expérimentales :

* la longueur d'onde du rayonnement,

* sa polarisation éventuelle,

* la durée de l'impulsion,

* les densités de puissance et d'énergie au niveau de la surface,

* la taille de la zone d'interaction,

* l'angle d'incidence du rayonnement par rapport à la surface,

* la nature et la pression du gaz ambiant ou du gaz de couverture

- Les facteurs liés au matériau :

* la nature,

* les caractéristiques microstructurales,

* l'état de surface initial, en termes de topographie, propriétés mécaniques, composition chimique.

De nombreuses études ont déjà été effectuées sur ce sujet đu fait de la diversité des applications (usinage, découpe, soudage, traitement de surface, élaboration de films par ablation laser, traitement par choc, densification etc....) au moyen de lasers émettant dans le visible $(0,53 \mu \mathrm{m})$ le proche infrarouge $(1,06 \mu \mathrm{m})$ ou l'infrarouge moyen $(10,6 \mu \mathrm{m})$. 
Ces études ont mis en évidence leurs caractères essentiels pour la mise au point et le transfert de ces nouveaux procédés laser vers les industries civiles et de défense.

Par contre, beaucoup moins de travaux ont été réalisés au moyen de sources émettant dans l'ultraviolet. Les résultats présentés dans cette communication rendent compte d'expériences, menées au moyen de lasers à exciplexes émettant à $248 \mathrm{~nm}$ et $308 \mathrm{~nm}$ pour améliorer la compréhension et le contrôle des processus fondamentaux (optique, thermique, mécanique, hydrodynamique, radiatif, métallurgique, physicochimique,...) mis en jeu lors de l'interaction entre un rayonnement de forte densité de puissance $\left(>\mathrm{MW} / \mathrm{cm}^{2}\right)$ et des matériaux métalliques et céramiques.

L'objectif de ce travail est principalement l'amélioration des propriétés tribologiques, des propriétés mécaniques, des propriétés physico-chimiques et l'obtention d'une meilleure tenue à la corrosion en milieu aqueux ou à l'oxydation à haute température.

Les expériences ont été réalisées au moyen de différentes sources laser : deux sources laser à exciplexes $\mathrm{KrF}$, l'une impulsionnelle, mono impulsion de forte énergie "KIR" (200 J, $400 \mathrm{~ns}$ ) permettant de grandes surfaces d'irradiation, la deuxième à fonctionnement impulsionnel répétitif de faible puissance moyenne "Lambda Physik $\mathrm{X}$ $220 \mathrm{i}^{\prime \prime}(80 \mathrm{~W}, 200 \mathrm{~Hz}, 20 \mathrm{~ns})$ ainsi que d'un laser $\mathrm{XeCl}$ Siemens $(40 \mathrm{~W}, 20 \mathrm{~Hz}, 40 \mathrm{~ns})$, lasers permettant de se rapprocher du procédé industriel.

Les essais ont été effectués pour ce qui concerne les métaux sur des alliages à base d'aluminium 2017 A (couramment appelé AU4G ou Duralumin) et à base de titane Ti.6A1.4V (TA6V) ainsi que sur des aciers 35NCD16 ou $316 \mathrm{~L}$. Les échantillons ont été préparés de façon à obtenir un même état de propreté (éthanol + ultrasons), une rugosité de surface parfaitement calibrée (polie $\mathrm{Ra}<0,5 \mu \mathrm{m}$; rugueuse $0,5<\mathrm{Ra}<10 \mu \mathrm{m}$ ). Pour ce qui concerne les céramiques, alumine $\mathrm{Al}_{2} \mathrm{O}_{3}$, nitrure d'aluminium $\mathrm{AlN}$, carbure de silicium SIC et zircone $\mathrm{ZrO}_{2}$ ont été testés.

Les matériaux ont été irradiês sous des conditions expérimentales choisies volontairement très différentes : - large gamme de densité d'énergie comprise entre 0,3 et $150 \mathrm{~J} / \mathrm{cm}^{2}$ soit 1 à $500 \mathrm{MW} / \mathrm{cm}^{2}$;

-conditions de pressions ambiantes, pression atmosphérique, atmosphère raréfié $\left(10^{+5}\right.$ à $10^{-2} \mathrm{~Pa}$ ) ;

- diversité de nature des gaz environnants tels que air, argon, azote, hélium.

Les principaux résultats concernent :

I. Modification de la topographie de la surface - amélioration des propriétés tribologiques

Ce type de traitement peut s'appliquer à la "préparation des surfaces" en vue de collage ultérieur, ou de diminution des effets de frottements. L'évolution de la rugosité a été étudiée expérimentalement en vue de comprendre, contrôler et quantifier la modification de la morphologie de la surface. Cette modification peut être de deux types; on peut constater d'une part une réduction de la rugosité (effet de lissage) par chauffage, fusion et vaporisation préférentielles sur les discontinuités de surface (rayures de polissage, inclusions, impuretés...) dans le cas où la rugosité initiale est de plusieurs microns (Fig. 1).

Dans le cas réel, une pièce métallique utilisée en frottement verra ses propriétés augmentées si sa surface présente une rugosité réduite. De plus, il est courant d'observer la formation de cratères ( $10 \mu \mathrm{m}$ de taille caractéristique) autour d'inclusions dont la taille et la profondeur augmentent avec le nombre de tirs. Une application récente dans 
l'automobile consiste à former des cratères autour de sphérullites de graphite par irradiation UV de cylindres moteur. Ceci permet de diminuer le frottement chemise-piston en réalisant une réserve d'huile sur les parois et donc une lubrification nettement améliorée. Des tests ont été réalisés sur des automobiles en conditions d'utilisation routière. Une augmentation d'un facteur 3 du temps entre les vidanges a été mis en évidence, l'objectif étant à terme de n'utiliser qu'une charge d'huile pour $100000 \mathrm{~km}$.

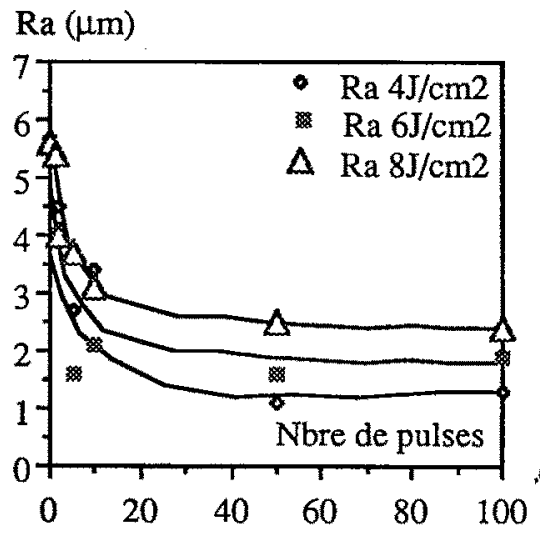

Fig. 1: Evolution expérimentale de la rugosité échantillon aluminium 2017A

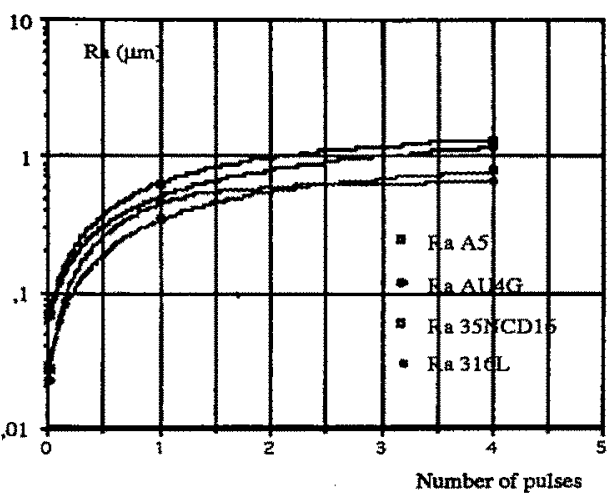

Fig.2: Evolution expérimentale de l'état de surface de différents matériaux; $F=80 \mathrm{~J} / \mathrm{cm}^{2}(300 \mathrm{~ns})$

D'autre part, si la rugosité initiale est relativement faible ( $\mathrm{Ra}<0,2 \mu \mathrm{m})$, on observe généralement une augmentation de la rugosité de surface après irradiation (Fig. 2). La formation de structures périodiques particulières (vaguelettes, "ripples", spirales,...) observées sur la surface fondue sont généralement attribuées aux instabilités hydrodynamiques de la surface liquide (Rayleigh-Taylor). Ces structures dépendent de nombreux paramètres liés aux conditions d'irradiation tels que la pression exercée par le plasma sur la surface, sa vitesse d'expansion au dessus du liquide, l'épaisseur du bain fondu, le gradient de température et la viscosité du métal liquide... La variation de la rugosité, plus importante lors des premiers tirs, semble se stabiliser lorsque le nombre d'irradiations augmente pour tendre vers une valeur quasi-stationnaire. En choisissant une rugosité initiale supérieure à cette limite, on obtient un lissage de la surface et en revanche, pour des Ra originaux inférieurs à cette limite, une augmentation systématique est observée. De plus, il est possible de modifier, dans une certaine limite, la topographie finale en jouant sur la densité d'énergie incidente. Il apparaît donc possible de contrôler la rugosité finale en choisissant judicieusement les paramètres laser.

Ces évolutions de la topographie de surface ont été analysées par profilométrie (Surfascan 3D) rugosimétrie mécanique (Talysurf 10) et visualisée par microscopie électronique à balayage (MEB JEOL JSM 6400).

Des essais réalisés à $308 \mathrm{~nm}, 40 \mathrm{~ns}$, sur différents matériaux tels que titane, acier alliages d'aluminium et de cuivre (1) ont montré qu'il était possible de corréler la modification de la surface avec la conductivité thermique. Si celle-ci est importante, la chaleur diffuse très rapidement en profondeur par conduction; le bain fondu par lequel le matériau est passé se fige très vite et reste rugueux du fait des effets de pression ou des instabilités hydrodynamiques de surface.

La figure 3 montre qu'il est plus aisé de lisser un échantillon de titane plutôt qu'un alliage à base d'argent. 


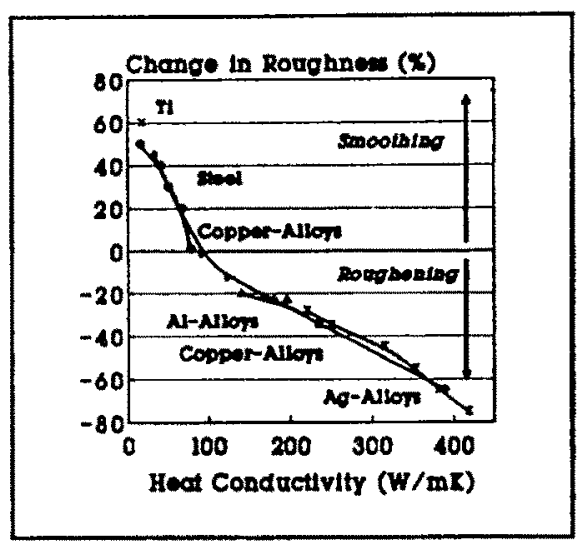

Fig. 3

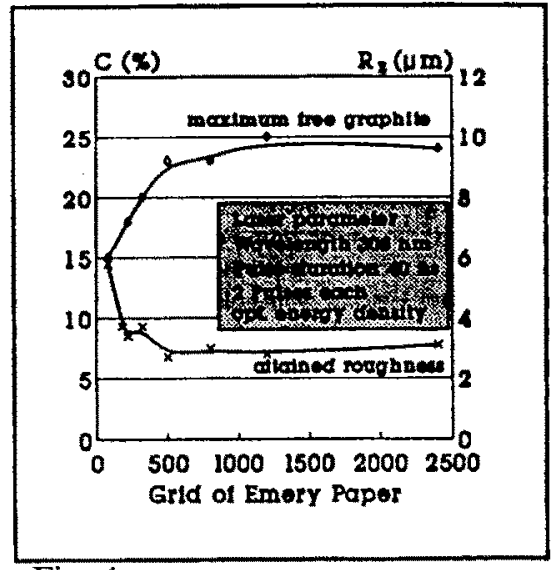

Fig. 4

La figure 4 présente l'évolution de la rugosité d'une fonte traitée au laser $(\mathrm{Rz}: 6-->3 \mu \mathrm{m})$ ainsi que le pourcentage de graphite en surface $(15->25 \mu \mathrm{m})$.

Pour ce qui concerne les céramiques, il est possible de relier la microstructure (taille des grains, porosité) avec les propriétés mécaniques et tribologiques (coefficient de frottement et usure en volume par exemple). La figure 5 met en évidence une augmentation importante du coefficient de frottement $(0,5 \rightarrow 1)$ d'une cible de zircone stabilisée à l'yttrium, $3 \% \mathrm{~mol} \mathrm{Y}_{2} \mathrm{O}_{3}-\mathrm{ZrO}_{2}$, lorsque la taille des grains varie de 0,3 à $1,1 \mu \mathrm{m}(2)$.

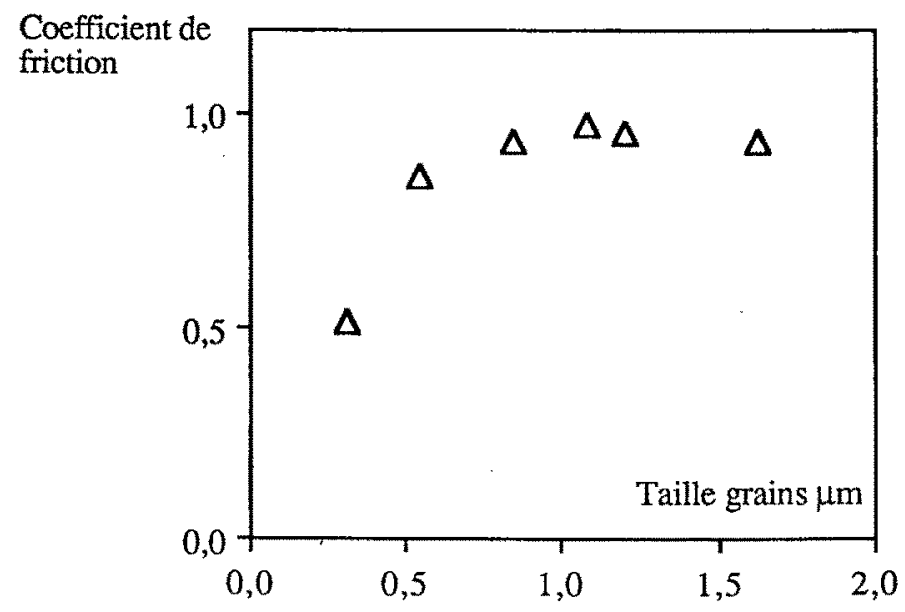

Fig. 5: Coefficient de friction de $\mathrm{ZrO} 2$ en fonction de la taille des grains

De même, l'usure totale sera plus importante avec la taille moyenne des grains de la structure: de 0,05 à $0,15 \mathrm{~mm}^{3} / \mathrm{km}$ pour 1 à $12 \mu \mathrm{m}$ pour $\mathrm{Al}_{2} \mathrm{O}_{3}$ et de 5 à 40 pour 0,3 à $1,6 \mu \mathrm{m}$ pour $\mathrm{ZrO}_{2}$, d'où l'intérêt de modifier la surface d'une céramique (rugosité, porosité) par refusion superficielle des grains (figure 6 et 7 , référence ${ }^{(3)}$ ). Les applications peuvent être intéressantes pour des pièces de roulements, des sièges de soupapes, des outils de découpe, des prothèses de hanche,... . 


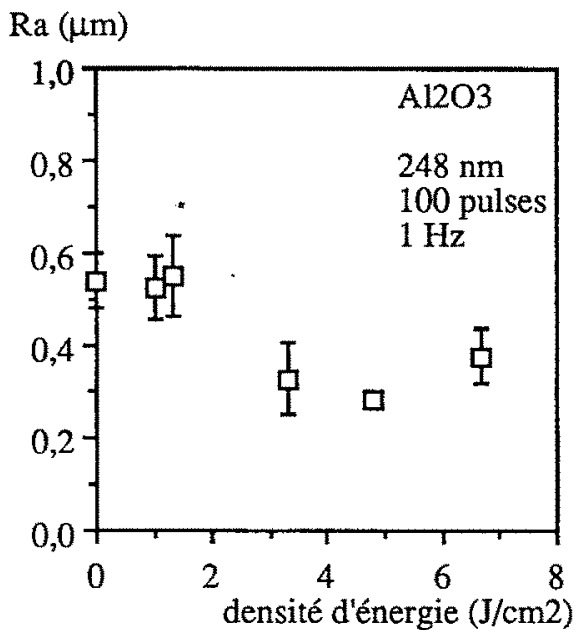

Fig. 6 Diminution de la rugosité sur $\mathrm{Al}_{2} \mathrm{O}_{3}$

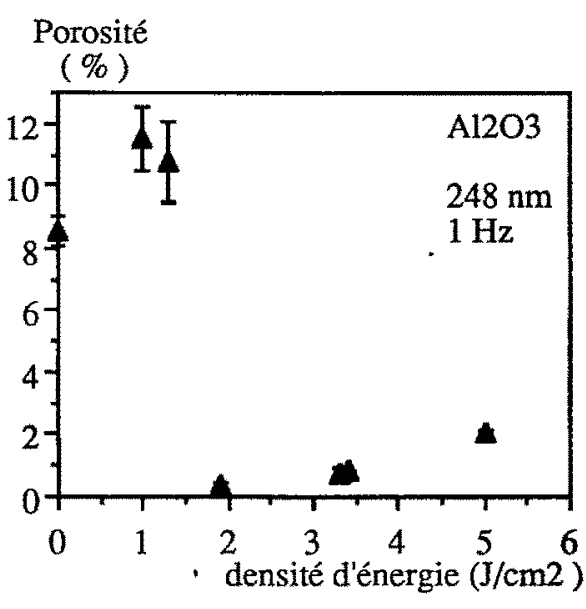

Fig. 7 Diminution de la porosité

II. Modification des propriétés mécaniques

Si on s'intéresse aux propriétés mécaniques d'un matériau, il faut prendre en compte les changements de dureté, les contraintes résiduelles induites dans la masse pour des éventuelles améliorations de la résistance à la flexion, à la traction ou à la fatigue.

a) Dureté

Des mesures de dureté des surfaces traitées ont été réalisées au moyen d'un microduromètre (Leitz Vickers indenter) chargé de 10 et $100 \mathrm{~g}$. Des variations de đureté significatives (4) ont été relevées dans le sens d'une augmentation pour les échantillons d'acier et de titane, et d'une diminution pour l'aluminium A5 et l'AU4G.

Dans le cas du 35 NCD16, l'augmentation de la dureté superficielle (200 HV 10 -$\rightarrow>900 \mathrm{HV}$ 10) par rapport au matériau vierge peut être corrélée avec la formation d'une fine couche de matériau fondu à structure martensitique, structure observable par microscopie électronique à transmission et X rasants (Fig.8).

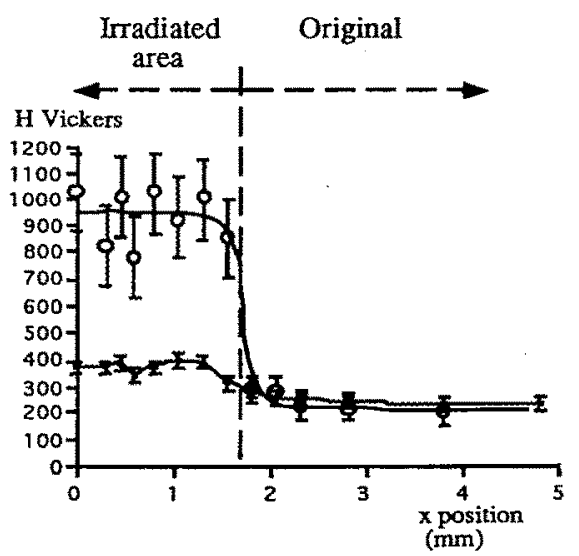

- Charge 10g

- Charge $100 \mathrm{~g}$

Fig.8: Test de dureté sur 35NCD16; $350 \mathrm{MW} / \mathrm{cm}^{2}, 300 \mathrm{~ns}$ 
Dans le cas d'un alliage d'aluminium, la décroissance peut résulter d'un recuit thermique de la surface avec formation de précipités particuliers $\mathrm{AlCu}_{2}$ par exemple (Cf. paragraphe III). Des microfissures ont également été détectées grâce aux observations au MEB. Des coupes transversales mettent en évidence des épaisseurs affectées thermiquement de $3 \mu \mathrm{m}$ pour l'acier, $7 \mu \mathrm{m}$ pour le titane, $12 \mu \mathrm{m}$ pour l'AU4G. La tenue au frottement et à l'usure du titane peut être aussi augmentée grâce à la formation d'une couche superficielle composée de nitrure de titane TiN, nitrure d'aluminium AlN et nitrure de vanadium VN. Des mesures réalisées par diffraction de rayons $X$ rasants laissent apparaître, par superposition des diffractogrammes, des pics caractéristiques de TiN, AlN et VN. Ces pics sont systématiquement déplacés par rapport aux raies des fiches JCPDS, révélant ainsi la présence d'une couche resolidifiée non-stoechiométrique de ( $\mathrm{Ti}, \mathrm{Al}, \mathrm{V}) \mathrm{N}_{1-\mathrm{x}}$. Des tests d'usure réalisés en Allemagne sur des chemises de moteur en fonte ont montré une augmentation de la durée de vie des pièces ; ceci est expliqué (1) par la présence d'azote en proportion non négligeable $(\approx 10 \%)$ dans la couche fondue formant ainsi une couche protectrice nitrurée.

b) Mesures des contraintes résiduelles

Des analyses, en surface et en profondeur (Fig.9 et Fig.10), au moyen d'un microscope à diffraction de rayons X (SET-X) d'échantillons de 35 NCD 16 , détensionné à l'origine, ont mis en évidence un champ important de contraintes résiduelles de traction, ceci malgré son augmentation de dureté(4). Cette relaxation des contraintes de compression par effet thermique (traitement en phase liquide) correspond à une diminution de la tenue en fatigue du matériau. Cet inconvénient peut être annulé dans le cas d'un traitement par choc laser.

Les échantillons recouverts d'un revêtement absorbant et facilement vaporisable sont irradiés à très haute densité de puissance à travers un diélectrique transparent, l'objectif étant de générer de fortes pressions dans le solide. Des expériences réalisées à $1,06 \mu \mathrm{m}$ ont permis d'obtenir des champs de contraintes résiduelles de compression sur des grandes surfaces et en profondeur.

D'après une étude bibliographique sur l'acier 35 NCD 16 , il est possible d'affirmer que, si la densité d'énergie augmente, des caractéristiques topographiques de la surface ( $\mathrm{Ra}, \mathrm{Rz}, \mathrm{Sm}$ etc....) et l'épaisseur de la zone fondue vont augmenter; de même l'amplitude des contraintes résiduelles surfaciques. En revanche, la finesse de la microstructure, le gradient des contraintes ainsi que le pourcentage de transformation en acier martensitique évoluera de façon inversement proportionnelle à la densité d'énergie déposée.

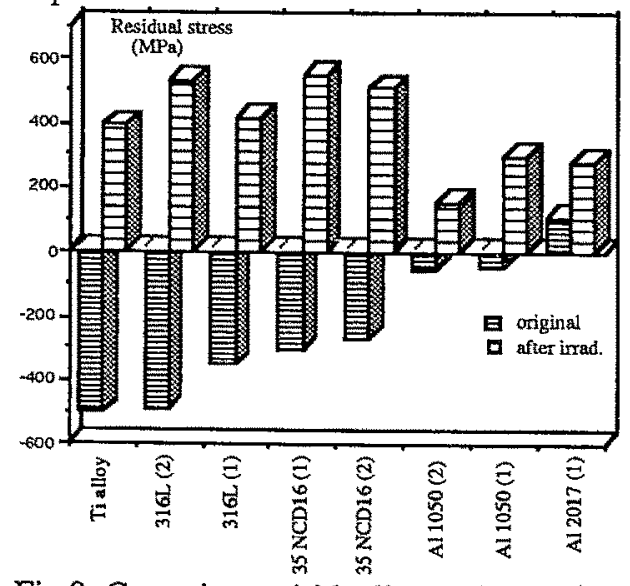

Fig.9: Contraintes résiduelles surfaciques $(200 \mathrm{MW} / \mathrm{cm} 2 ; 300 \mathrm{~ns})$

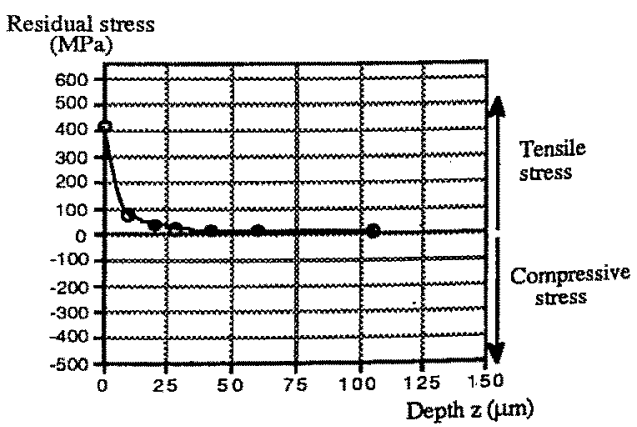

Fig.10: Profil de contraintes dans un acier 35NCD16, irradiation directe 
D'une façon générale, suivant les conditions de traitement, les contraintes résiduelles, microstructure, topographie et épaisseur affectée sont des paramètres étroitement liées.

c) Amélioration de la tenue à la flexion d'une céramique:

Des traitements de surface sur alumine au moyen d'un laser $\mathrm{KrF}$ à $3 \mathrm{~J} / \mathrm{cm} 2$ ont mis en évidence une modification du module de Weibull (1) avec une augmentation d'un facteur 2. Ce module, inversement proportionnel à la probabilité de rupture a été déterminé pour différents qualités d'alumine (Fig 11).

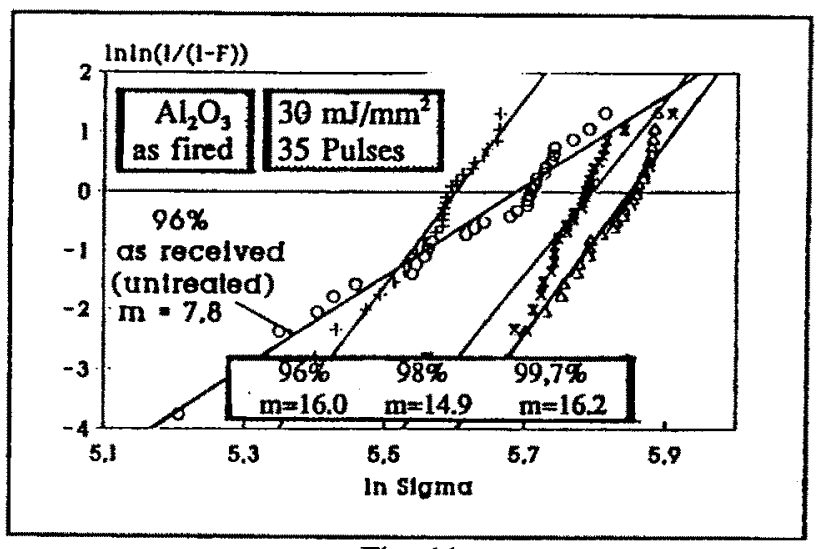

Fig. 11

III. Modification des propriétés physico-chimiques- Amélioration de la tenue à la corrosion

Des modifications de la composition chimique ont été observées sur le titane par exemple par obtention d'une couche protectrice nitrurée (TiAlV) $\mathrm{N}_{1-\mathrm{x}}$ précédemment évoquée, sur l'alliage d'aluminium par la formation de $\mathrm{Al}_{2} \mathrm{Cu}$, précipité protecteur contre l'oxydation (khatyrkite).

De plus, la surface d'un alliage $\mathrm{Ni}-\mathrm{Cr}$, ou d'un acier inox, traitée laser peut s'enrichir en chrome par migration d'atomes, sa tenue à la corrosion en milieu aqueux se trouvant ainsi nettement améliorée.

La diminution importante de la porosité après traitement de $\mathrm{Al}_{2} \mathrm{O}_{3}(12->1 \%)$ à 2$3 \mathrm{~J} / \mathrm{cm} 2$ à $248 \mathrm{~nm}$ (3) contribue également à améliorer le comportement d'un matériau à structure granulaire face aux agressions aqueuses ou gazenses à haute température. Un revêtement en zircone dans un moteur diesel ou turbine à gaz, par exemple; peut subir une fusion superficielle suivie d'une solidification rapide conduisant à une densification de la surface et devenir ainsi plus étanche aux gaz corrosifs (5).

\section{Modification des propriétés électriques}

Une autre possibilité de traitement de ces céramiques, AlN par exemple, est la gravure directe par irradiation laser, de pistes conductrices à la surface du matériau massif, par ablation d'azote et enrichissement en métal (aspect brillant). La céramique se comporte alors comme un semi- conducteur. Des estimations de résistivité ont été obtenues et varient de $10^{-4} \Omega$.cm pour un traitement à $6 \mathrm{~J} / \mathrm{cm} 2(248 \mathrm{~nm}, 10 \mathrm{tirs}, 10 \mathrm{~Hz})$ à $10^{10} \Omega$.cm pour un échantillon non-traité (6). Dans le cas où la densité d'énergie est trop importante, le matériau conducteur peut être ablaté et la résistance augmenter à nouveau. 


\section{Conclusion}

Les travaux réalisés dans le cadre de cette étude ont montré qu'il était possible, dans des conditions d'irradiation appropriées de modifier certaines caractéristiques des matériaux métalliques et céramiques, principalement utilisés dans le domaine de l'automobile et de l'espace. De nombreuses investigations restent à faire pour établir des relations entre la microstructure des matériaux traités et leurs propriétés mécaniques.

\section{Remerciements}

Les analyses métallurgiques et tests de rugosité sur les céramiques ont été en partie réalisées dans le Laboratoire de Mécanique des Surfaces de l'ENSAM à Aix en Provence. Les auteurs remercient les responsables et le personnel de ce laboratoire qui ont permis l'obtention de ces résultats.

\section{Références}

1. H. W Bergmann, E. Shubert, K. Schutte, "Surface modifications using excimer lasers; fundamentals and applications. Journal de physique IV, Vol. 1, 7-12, décembre 1991.

2. K. H. Zumgahr, W. Bundschuh, B. Zimmerlin, "Effect of grain size on friction and sliding wear of oxide ceramics"; Wear 162-164, pp 269-279, 1993.

3. C. Prat, G. Nicolas Costa, M. Autric, "Caractérisation de matériaux céramiques soumis à rayonnement laser UV. Applications au traitement de surface. UVX 94. 13-16 juin 1994.

4. T. Sarnet, J-E. Montagne, G. Inglesakis, M. Autric, L. Barrallier, G. Barreau, "Modification of metal by high energy excimer laser", Journal de Physique JPIV, Vol. 4, pC4-C9, 1994.

5. A. Petitbon, "Traitements par laser de revêtements céramiques", Laser de puissance et traitement des matériaux, pp 611-620, 1991.

6. A Tsetsekou, Th. Zambetakis et al. "Excimer laser treatment of aluminium nitride". SPIE Vol. 1810, GCL 615-619, 1992. 